\title{
An assessment of data and methodology of online surgeon scorecards
}

\author{
Linda W. Xu, MD, ${ }^{1}$ Amy Li, BA, ${ }^{1}$ Christian Swinney, BA, ${ }^{1}$ Maya Babu, MD, MBA, ${ }^{2}$ \\ Anand Veeravagu, MD, ${ }^{1}$ Stacey Quintero Wolfe, MD, ${ }^{3}$ Brian V. Nahed, MD, MSc, ${ }^{4}$ and John K. \\ Ratliff, MD, ${ }^{1}$ in affiliation with the Council of State Neurosurgical Societies
}

1Department of Neurosurgery, Stanford University School of Medicine, Palo Alto, California; ${ }^{2}$ Department of Neurosurgery, Mayo Clinic, Rochester, Minnesota; ${ }^{3}$ Department of Neurosurgery, Wake Forest University School of Medicine, Winston-Salem, North Carolina; and ${ }^{4}$ Department of Neurosurgery, Massachusetts General Hospital, Boston, Massachusetts

OBJECTIVE Recently, 2 surgeon rating websites (Consumers' Checkbook and ProPublica) were published to allow the public to compare surgeons through identifying surgeon volume and complication rates. Among neurosurgeons and orthopedic surgeons, only cervical and lumbar spine, hip, and knee procedures were included in this assessment.

METHODS The authors examined the methodology of each website to assess potential sources of inaccuracy. Each online tool was queried for reports on neurosurgeons specializing in spine surgery and orthopedic surgeons specializing in spine, hip, or knee surgery. Surgeons were chosen from top-ranked hospitals in the US, as recorded by a national consumer publication ranking system, within the fields of neurosurgery and orthopedic surgery. The results were compared for accuracy and surgeon representation, and the results of the 2 websites were also compared.

RESULTS The methodology of each site was found to have opportunities for bias and limited risk adjustment. The end points assessed by each site were actually not complications, but proxies of complication occurrence. A search of 510 surgeons (401 orthopedic surgeons [79\%] and 109 neurosurgeons [21\%]) showed that only $28 \%$ and $56 \%$ of surgeons had data represented on Consumers' Checkbook and ProPublica, respectively. There was a significantly higher chance of finding surgeon data on ProPublica $(p<0.001)$. Of the surgeons from top-ranked programs with data available, $17 \%$ were quoted to have high complication rates, $13 \%$ with lower volume than other surgeons, and $79 \%$ had a 3 -star out of 5 -star rating. There was no significant correlation found between the number of stars a surgeon received on Consumers' Checkbook and his or her adjusted complication rate on ProPublica.

CONCLUSIONS Both the Consumers' Checkbook and ProPublica websites have significant methodological issues. Neither site assessed complication occurrence, but rather readmissions or prolonged length of stay. Risk adjustment was limited or nonexistent. A substantial number of neurosurgeons and orthopedic surgeons from top-ranked hospitals have no ratings on either site, or have data that suggests they are low-volume surgeons or have higher complication rates. Consumers' Checkbook and ProPublica produced different results with little correlation between the 2 websites in how surgeons were graded. Given the significant methodological issues, incomplete data, and lack of appropriate risk stratification of patients, the featured websites may provide erroneous information to the public.

https://thejns.org/doi/abs/10.3171/2016.7.SPINE16183

KEY WORDS orthopedic surgery; surgeon ratings; Consumers' Checkbook; ProPublica; website

I N July 2015, 2 surgeon rating websites, Consumers' Checkbook and ProPublica, were released to the public. The goal of each site was to provide patients with the surgical outcomes for common elective surgeries considered relatively low risk based on data released from the Centers for Medicare and Medicaid Services (CMS). ${ }^{25}$
These 2 websites estimate surgeon case volume and complication rates, grading surgeons against their colleagues. Providing patients with easy online access to performance data of surgeons is one means to allow patients to make more informed decisions. Performance metrics are increasingly used by hospitals and surgeons to promote bet-

ABBREVIATIONS CABG = coronary artery bypass grafting; $\mathrm{Cl}=$ confidence interval; CMS = Centers for Medicare and Medicaid Services; LOS = length of stay; NSQIP = National Surgical Quality Improvement Program.

SUBMITTED February 11, 2016. ACCEPTED July 11, 2016.

INCLUDE WHEN CITING Published online September 23, 2016; DOI: 10.3171/2016.7.SPINE16183. 
ter practices and outcomes. Press releases heralding the release of the websites offered identification of individual surgeons with higher than expected complication occurrences. The implication was that these named surgeons were providing lower quality care than their colleagues.

There are critiques of these approaches that have been reported. A methodological critique of the ProPublica Surgeon Scorecard was recently published by Friedberg et al. at RAND Health, which highlighted potentially significant problems in the way ProPublica chose to rate surgeons. These problems include issues with their "adjusted complication rates," which do not incorporate complications occurring without readmission, during the index hospitalization (when the surgery took place), or beyond the 30 days postdischarge. ${ }^{8}$ In addition, ProPublica ignores hospital-to-hospital variation in their analysis, assuming instead that the surgeon performs at a "hypothetical average hospital," which may undermine comparisons between physicians operating in different facilities. ${ }^{8,24}$

Both Consumers' Checkbook and ProPublica have created surgeon rating algorithms based on methodologies that are not peer reviewed and have not been subject to appropriate scientific scrutiny. While there have been critiques of these methods, no study has quantitatively analyzed the ratings produced by these websites. In this study, we aim to both qualitatively review the methods in which surgeons are rated and quantitatively determine the utility of these surgeon rating websites when used to assess a group of surgeons in the fields of spinal neurosurgery and orthopedic surgery.

\section{Methods \\ Methodology Assessment}

We reviewed the methodology of each site via their published methods sections. Appendices outlining risk adjustment strategies, when present, were reviewed. We relied on reporting by the individual sites on their publically accessible websites for methodology descriptions.

\section{Hospital Selection}

To identify a set of surgeons for this assessment, we selected spinal neurosurgeons and orthopedic surgeons from the top 20 hospitals as designated by the 2015-2016 US News and World Report in Neurology \& Neurosurgery and Orthopedics, respectively. Review of only large, top-rated institutions helped standardize our comparison. While our findings may translate into the community setting as well, our study design, which omitted surgeons at smaller centers for the sake of standardization, prevents this from being definitively concluded.

In neurosurgery, the following 20 hospitals were included in our analysis: Mayo Clinic, Massachusetts General Hospital, Johns Hopkins Hospital, UCSF Medical Center, New York-Presbyterian University Hospital of Columbia and Cornell, Brigham and Women's Hospital, UCLA Medical Center, Cleveland Clinic, NYU Langone Medical Center, Northwestern Memorial Hospital, Barnes-Jewish Hospital/Washington University, Emory University Hospital, Hospitals of the University of Pennsylvania-Penn Presbyterian, Mount Sinai Hospital, Rush University
Medical Center, University of Pittsburgh Medical Center, Cedars-Sinai Medical Center, University of Kansas Hospital, IU Health Academic Health Center, and St. Joseph's Hospital and Medical Center. We excluded Houston Methodist Hospital (ranked no. 16) in this study because the hospital did not specify spine specialty neurosurgeons on their website. We replaced this with St. Joseph's Hospital and Medical Center (ranked no. 21) in our analysis.

In orthopedic surgery, the following 20 hospitals were included: Hospital for Special Surgery, Mayo Clinic, Cleveland Clinic, Massachusetts General Hospital, Hospital for Joint Diseases (NYU Langone Medical Center), Rush University Medical Center, Brigham and Women's Hospital, Santa Monica-UCLA Medical Center and Orthopedic Hospital, Northwestern Memorial Hospital, New England Baptist Hospital, University of Pittsburgh Medical Center, Cedars-Sinai Medical Center, UCSF Medical Center, Thomas Jefferson University Hospital, University of California Davis Medical Center, Beaumont Hospital, Barnes-Jewish Hospital/Washington University, University of Iowa Hospital and Clinics, Abbott Northwestern Hospital, and Johns Hopkins Hospital.

\section{Neurosurgeon and Orthopedic Surgeon Selection}

From the top 20 hospitals in the 2015-2016 US News and World Report, we identified spinal neurosurgeons and orthopedic surgeons. The methodology of Consumers' Checkbook (http://www.checkbook.org/surgeonratings) and ProPublica (https://projects.propublica.org/surgeons) included only spine surgery and hip or knee replacement surgery. Therefore, we limited our selection of neurosurgeons to spine surgeons only; we included only orthopedic surgeons who specialized in spine, hip, or knee procedures. Specification of spine surgery instead of including general neurosurgeons was deemed necessary to maximize the accuracy of our findings. While the exact distribution of spine procedures cannot be definitively determined for such a large sample size, it can be assumed that those designated as spine surgeons primarily perform spine-related procedures. The same cannot be definitively said for general neurosurgeons.

Consumers' Checkbook only analyzed data sets of surgeons practicing between January 1, 2009, and December 31, 2012. ProPublica only examined surgeons between 2009 and 2013. To maximize the chances that our study surgeons would be included in both of the surgeon rating websites, we limited our selection of surgeons to those who had finished residency or fellowship no later than 2010. Data on year of residency graduation was obtained from each program's website. A total of 510 surgeons were included in this study, with 401 orthopedic surgeons (79\%) and 109 neurosurgeons (21\%).

\section{Consumers' Checkbook}

The Consumers' Checkbook website was searched for the individual surgeons' records between August 12, 2015, and August 23, 2015. The Consumers' Checkbook surgeon rating website provides the surgeon's full name, sex, location, hospital affiliations, contact information, and educational background, including board certification and 
medical school. The website assigns each surgeon a number of stars (3 to 5) based on how confident Consumers' Checkbook is of the surgeon's "better-than-average" outcomes. A 5-star rating represents the highest confidence that the surgeon's results are better than that of the average surgeon in the US. A 3-star rating represents the lowest confidence of the surgeon's outcomes. When a surgeon is not given stars, it is either because the surgeon's results are not better than average or there are not enough data on the surgeon. Hence, no surgeon is awarded fewer than 3 stars. In patients without a star rating, the website does not distinguish between lack of data or data indicating that the surgeon's results are below or equivalent to average. Consumers' Checkbook also rates the surgeon's operative volume compared with other doctors in their database: the volume of surgeries is listed as lower, medium, or higher volume.

\section{ProPublica}

The ProPublica website was searched for the individual surgeons' records between August 12, 2015, and August 23,2015 . The ProPublica surgeon rating website provides the following output for each surgeon: surgeon's full name, hospital affiliation, and complications related to the types of surgery performed. ProPublica provides the number of times the surgeon performed each operation, the reported number of complications, and the adjusted complication rate. The adjusted complication rate is further divided into low, medium, and high in comparison with other surgeons for the same operation. In the event of multiple operations listed, the adjusted complication rates were averaged for each surgeon.

\section{Statistical Analysis}

Data for each selected surgeon were collected from each website. The total number of operations performed was calculated by adding separate surgical types listed on ProPublica. An average complication rate was calculated using the separate complication rates listed per procedure. A chi-square test was used to compare availability of data between the 2 websites. A Student t-test was used to compare complication rates on ProPublica and star rating on Consumers' Checkbook, and estimates of surgeon volume between websites.

\section{Results}

\section{Consumers' Checkbook}

Using CMS inpatient data from January 1, 2009, to December 31, 2012, adverse outcomes following 18 surgical procedures were recorded for each surgeon from the Consumers' Checkbook website. Adverse outcomes were defined as inpatient mortality, prolonged risk-adjusted length of stay (LOS), 90-day postdischarge mortality, and 90-day readmission. Logistic regression was used to estimate the probability of an adverse outcome and used to adjust the measured adverse outcome rate to create a risk-adjusted rate for each surgeon. This logistic regression included considerations for age, sex, year of the procedure, certain selected other diagnostic codes the patient also carried, and combinations with other procedures at the time of intervention. The algorithms of how prolonged risk-adjusted
LOS or the logistic regression are calculated are not provided by Consumers' Checkbook for review.

\section{ProPublica}

Using CMS inpatient data from 2009 to 2013, adverse outcomes following 8 surgical procedures were recorded for each surgeon from the ProPublica website. Complications were defined as death during initial hospital stay and 30-day readmission for a principal diagnosis suggestive of negative surgical outcome by a panel of reviewers. Complications were then adjusted for using logistic regression models for age, sex, health score based on comorbidities, and multilevel spinal procedures for spine surgeries.

\section{Issues in Consumers' Checkbook and ProPublica Methodology}

Both surgeon rating websites claim to provide surgical outcomes for each surgeon in comparison with his or her colleagues: Consumers' Checkbook in the form of a star grading system, and ProPublica in the form of their adjusted complication rate classification. However, neither website calculates complications through accepted standards by surgeons or the general public. . $^{171,27}$

Consumers' Checkbook uses the term "complications" but does not actually measure true surgical complications, instead using LOS, readmissions, and deaths as proxies for occurrence of complications after surgery. ${ }^{4}$ ProPublica uses the term "complications" when they are really referring to readmissions to the hospital and death. ${ }^{24}$ The literature reports a high number of complications occurring during the patient's initial hospitalization rather than subsequent patient encounters. ${ }^{3,18,32,34,35,38,39}$ For readmissions, the ProPublica site reviewed only the principal diagnosis as the cause for the readmission. Approximately half of readmissions in the ProPublica effort were considered to be secondary to postoperative complications. The site does not capture complications that occurred after a procedure during a patient's initial, index hospitalization or complications that are managed on an outpatient basis.

Consumers' Checkbook also does not directly assess perioperative complications, only examines LOS as compared with predicted LOS, examines only 90-day mortality, and uses prolonged LOS as a proxy for complication occurrence. The site does not explain how it developed a predicted LOS for each patient and does not exclude other reasons for prolonged LOS that may not be related to surgeon factors or occurrence of perioperative adverse events.

\section{Risk Adjustment}

Consumers' Checkbook reports using a risk-adjusted LOS to measure surgeon outcomes, but they were vague about the details used to create the risk adjustment. ${ }^{5}$ ProPublica uses a "health score," which is a modification of the Elixhauser comorbidity score, as an indicator of preoperative risk. ${ }^{24,33}$ This measure was developed to combine the Charlson comorbidity system with the Elixhauser classification system to measure a patient's medical risk for in-hospital deaths. ${ }^{33}$ The Charlson comorbidity score may not be a reliable predictor of complication risk, especially in patients undergoing spine surgery. ${ }^{36}$ 
Using the described "health score" as a predictor of complications in surgical patients is not confirmed in the literature. ProPublica found little merit in their risk-adjustment approach: there was minimal correlation between a patient's health score and postoperative complication occurrence, with an area under the curve of $0.57-0.63$ per procedure. ${ }^{24}$

\section{Consumers' Checkbook Scoring}

We searched a total of 510 surgeons in the Consumers' Checkbook surgeon rating website: 401 orthopedic surgeons (79\%) and 109 neurosurgeons (21\%). Consumers' Checkbook only found information on 145 surgeons (28\%), with $72 \%$ of surgeons having no surgical information or listing on the website. Two surgeons had a profile but no stars listed. Of the surgeons found, 21 (14\%) had a 5-star rating, 10 (7\%) had a 4-star rating, and 114 (79\%) had the lowest 3 -star rating. The average number of stars assigned was 3.36. Concerning operative volume, 105 surgeons $(73 \%)$ had a higher volume, 20 surgeons (14\%) had a medium volume, and 18 surgeons (13\%) had a lower volume of surgeries compared with other doctors in the same field.

\section{ProPublica Scoring}

We searched the same 510 surgeons in the ProPublica surgeon rating website. ProPublica had an entry for 413 surgeons $(81 \%)$ on the website, but only $286(56 \%)$ of these surgeons had any surgical data listed in their profile. The median number of surgeries listed per surgeon with data was 119 (range 20-1546 surgeries). The median adjusted complication rate was 2.8 (range 1.2-7.5 complications). Of the surgeons with data, different complication rates were given per elective procedure examined: 25 surgeons (9\%) had a low adjusted complication rate, $270(94 \%)$ a medium adjusted complication rate, and $50(17 \%)$ a high adjusted complication rate for at least 1 listed procedure.

\section{Comparing the Websites}

The median complication rate of $2.8 \%$, ranging from $1.2 \%$ to $7.5 \%$, likely vastly underestimates the true complication rate after spine and joint surgeries compared with published series of $1 \%-10 \%$ of morbidity for spine procedures and up to 7\%-9\% for hip and knee replacements. $67,12,13,15,19,20$ Ninety-four surgeons (18.4\%) were not represented on either surgeon rating website. The relative likelihood of finding a surgeon's name on ProPublica was 2.8 times higher than Consumers' Checkbook (95\% confidence interval $[\mathrm{CI}] 2.5-3.3, \mathrm{p}<0.001)$. The relative risk of finding a surgeon's data (not just the name) on ProPublica was 2.4 times higher than Consumers' Checkbook $(95 \%$ CI $2.1-2.8, \mathrm{p}<0.001$ ).

We compared ProPublica's adjusted complication rate categories and Consumers' Checkbook's outcome grading ( 3,4 , or 5 stars) to analyze how well the 2 websites correlated with each other in evaluating the same surgeons. Surgeons with the lowest 3-star rating on Consumers' Checkbook had an average adjusted complication rate of $3.2 \%$ on ProPublica. Surgeons with a 4 -star rating were assigned an average of $3.1 \%$ for the adjusted complication rate on ProPublica. Finally, surgeons with the best 5-star rating on Consumers' Checkbook had a lower average adjusted complication rate of $2.6 \%$ on ProPublica. There was a trend toward decreasing complication rate as stars increased, but we found no statistical significance in the correlation ( $p=0.79$ between 3 and 4 stars, $p=0.23$ between 4 and 5 stars; Fig. 1).

We compared the operative volume estimated by both Consumers' Checkbook and ProPublica for surgeons. The actual number of cases listed on ProPublica was compared with the lower, medium, or higher volume classification given to surgeons on Consumers' Checkbook. There was a statistically higher average number of cases of "higher volume" surgeons compared with "medium volume" surgeons $(p=0.01)$. No significant difference in average cases reported on ProPublica existed between "medium volume" and "lower volume" surgeons ( $p=0.19$, Fig. 2).

\section{Discussion}

A valid assessment of surgeon-specific outcome requires high-quality input data, accepted definitions of complications and preoperative risks, and adequate sample sizes. ${ }^{2}$ The Consumers' Checkbook and ProPublica surgeon rating websites harbor severe methodological shortcomings with all 3 of these issues. The 2 sites claim to record surgeon complications and to offer a window into the clinical performance of surgeons nationwide; however, the output of these sites, as assessed in this study, is of limited value.

\section{Administrative Data}

The 2 sites use ICD-9 procedure codes to define their patient cohorts. There are substantial opportunities for

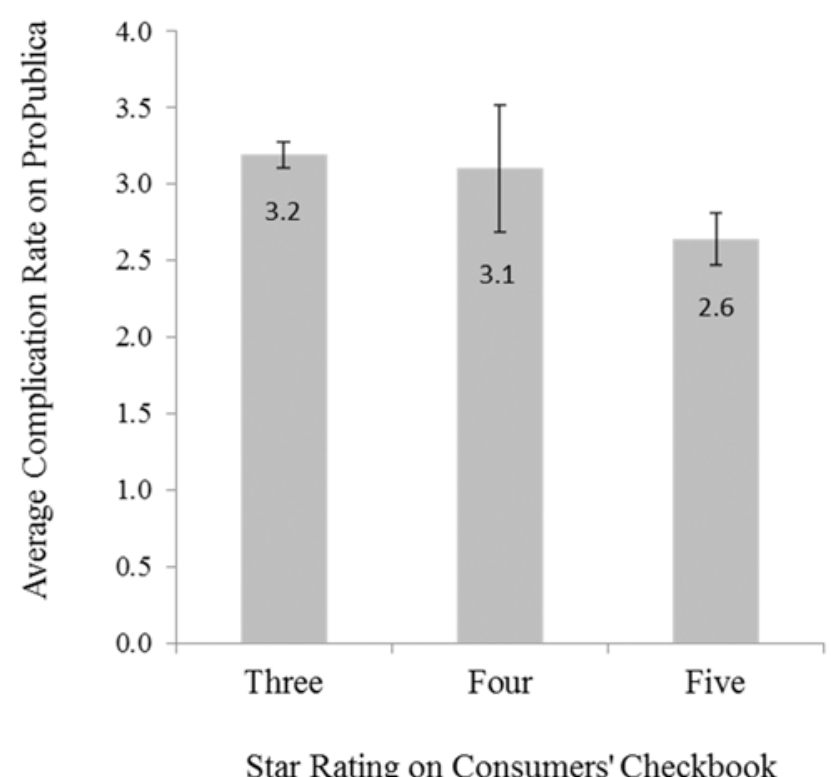

FIG. 1. Average adjusted complication rates given on ProPublica based on surgeon star rating on Consumers' Checkbook. The surgeons studied are spinal neurosurgeons and spinal, knee, or hip orthopedic surgeons. There was no statistically significant difference in the average complication rates between 3 - and 4-star ratings $(p=0.79)$ and between 4- and 5 -star ratings $(p=0.23)$. 


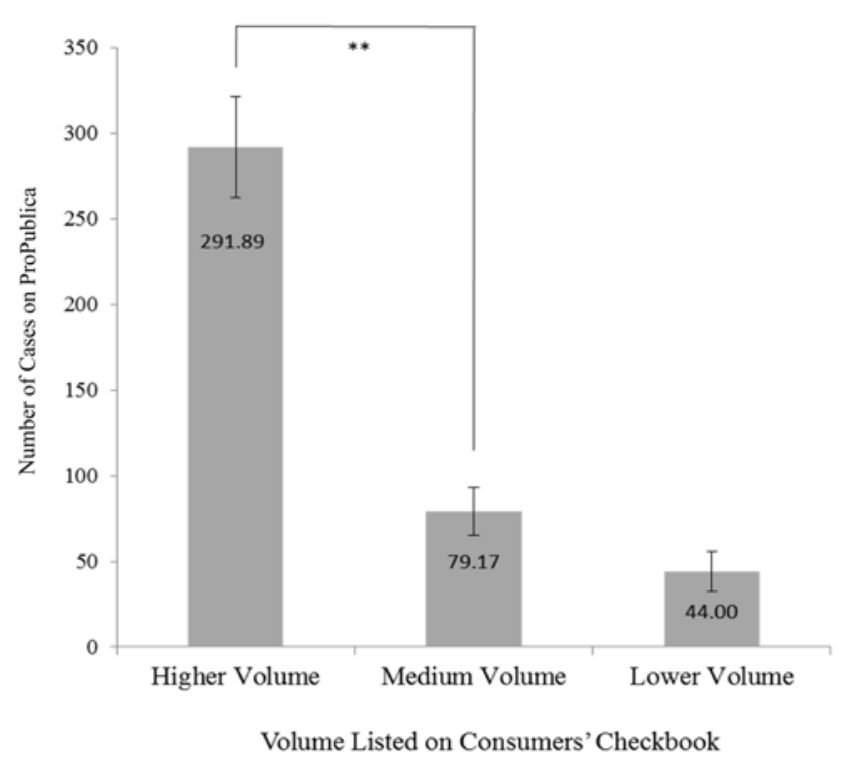

FIG. 2. A comparison of case volume on Consumers' Checkbook and number of cases on ProPublica for spinal neurosurgeons and spinal, knee, or hip orthopedic surgeons. ${ }^{* *} p=0.01$.

bias with this approach. The procedure codes combine a wide variety of procedures, with a wide variety of different complexities and different propensities for readmission risk. Spinal reconstructive procedures have a much higher risk of readmission and a higher risk of perioperative adverse event occurrence when compared with less extensive 1- or 2- level spinal fusions.

With this metric alone, surgeons performing reconstructive procedures would appear to have higher rates of complications/readmissions, and hence to have poorer results with this gross metric. Thus, for the spine surgery patient cohort, the 2 approaches actually analyze both low- and high-risk procedures. More than 2000 patients in the lumbar fusion (ICD-9 code 81.07) patient group are undergoing treatment with a primary diagnosis of scoliosis. For a Medicare-aged population, those procedures are likely reconstructive and not low risk. The researchers do not describe different complication rates in the different diagnosis categories they review.

\section{Definition of Complication and Risk Adjustment}

The definition of a complication used by each website is not representative of the experiences of surgeons and patients. A focus only on complications that produce a readmission or death likely misses the majority of complications occurring in this patient population. Neither website captures any other complications that occur without hospital readmission, perhaps the most common postoperative complications for elective spine or joint surgery. Use of prolonged LOS as a proxy for complication occurrence is not a validated approach and not a direct assessment of complication occurrence. Both websites provide inadequate information to patients who may consider complications of a procedure to be more than just those severe enough to render readmission, death, or a prolonged LOS. ${ }^{10,17,27}$
Focusing on complications that require readmission and on patient deaths in isolation may present a potentially significant sampling error that exposes these models to substantial bias. Ignoring complications that occur during a patient's index hospitalization likely ignores the majority of complications that a patient may suffer. The literature reports complication rates in lumbar fusion surgery conservatively of approximately $20 \%$, with higher rates in older patients and in patients with significant comorbidities. ${ }^{34,35}$ Some procedures may have complication rates of up to $50 \% ; 3,37,38$ this means the approximately $5 \%$ rate of readmissions focused on by the ProPublica report misses $75 \%$ or more of perioperative complications.

The ProPublica and Consumers' Checkbook methodologies offer poor or no risk adjustment in each surgeon assessment. Patient comorbidities and medical conditions are important predictors and confounders of complication occurrence after surgery and should be adjusted appropriately when measuring surgeon outcomes..$^{11,23,28,29}$ The "health score" reported as part of the ProPublica site has not been validated for use in spine surgery procedures and had low predictive accuracy. This poor predictive accuracy may be evidence of a weakness in the model's risk stratification; the ProPublica conclusion that patient comorbidities have no impact on complication occurrence is not supported by the literature. It has been suggested that logistic regression in general poorly identifies outliers and reduces variability observed from random noise, and that hierarchical models or multilevel modeling is a more statistically valid method, but no validated studies have used these models to assess real-world surgeon data. ${ }^{2,30}$

\section{Sample Size}

Deaths and readmissions in elective procedures are infrequent. Therefore, the rare readmission can drive a change in rating category for a surgeon, especially when both websites only account for data within a short 4-year (Consumers' Checkbook) or 5-year period (ProPublica). This small sample of readmitted patients may not be sufficiently powered to predict overall complication incidence and focuses on a small subset of severe events, not a surgeon's overall clinical care.

The small numbers of readmissions per surgeon mean that only a few readmissions may drive a change in score. The provided data for St. Joseph's Hospital in Phoenix, Arizona, reveal that 3 surgeons have high adjusted rates of readmissions/deaths. However, the overall number of readmissions/deaths for all surgeons from St. Joseph's is less than 10 per surgeon, and hence not reported. No raw readmission/death rate is reported for these surgeons. Since the total number of readmissions/deaths for each surgeon is redacted, further analysis is impossible. It is clear that, when low-, medium-, and high-risk surgeons are each having fewer than 10 readmissions/deaths over a 5-year data sample, perhaps only a single readmission could drive a change in risk category for a single surgeon. Statistically, a large number of procedures, possibly 2-10 times the average number of procedures performed by each surgeon, could be necessary to detect someone with true differences in complication rate. ${ }^{2}$ Thus, given these small sample sizes, the websites cannot truly predict which surgeons are either above or below average. 


\section{Findings for Surgeons From Top-Ranked Hospitals}

The flaws in the methodologies of both surgeon rating websites may explain the inconsistent representation of neurosurgeons and orthopedic surgeons from top-ranked hospitals in the US, as well as a lack of significant agreement in the grading of surgeons between websites.

In this study, we focused on how surgeons from topranked hospitals in the fields of neurosurgery and orthopedic surgery were represented in the 2 recently released surgeon rating websites. For Consumers' Checkbook, we found that $72 \%$ of surgeons were not represented on their website. For ProPublica, 19\% of the surgeons were not found on the website. Even among surgeons with entries in ProPublica by name, we saw no surgical data listed $31 \%$ of the time. Even among surgeons who had been practicing for years at top-ranked hospitals, only $9 \%$ had any procedures performed listed as low complication on ProPublica and $79 \%$ were listed as 3 stars, the lowest number of stars that can be listed on Consumer Checkbook.

We tested the internal validity between the 2 websites in evaluating the same surgeons. When displaying complication outcomes in comparison with their colleagues, ProPublica uses an adjusted complication rate and Consumers' Checkbook a star grading system. We found no statistical correlation between the number of stars a surgeon receives on Consumers' Checkbook and the surgeon's adjusted complication rate ranking on ProPublica. Operative volume estimates of surgeons between the 2 websites showed a greater correlation, but as only a few elective cases were included in evaluation of surgeon volume, this may not actually reflect true surgeon volume if the surgeon performs a great number of nonlisted procedures or nonelective cases.

The websites we assessed are advertised as being able to identify the best surgeons in the country compared with their peers. From a consumer perspective, an ideal surgeon would likely have low complication rates per ProPublica and high volume and 5 stars on Consumers' Checkbook. Of our list of the 510 surgeons in these fields, only 4 surgeons fit these criteria. Our data call into question how this information may be interpreted by consumers and whether it can truly be used to inform the choice of which surgeon to seek out for a procedure.

\section{Implications of Reporting Surgeon Outcomes}

Although it is difficult to predict how reported surgeon outcomes will affect the community of neurosurgeons and orthopedic surgeons, referring physicians, and patients, our best case study thus far is with cardiothoracic surgery, the first specialty to start public reporting. In the 1980s to 1990 s, a set of data outlining the surgical mortality rate following coronary artery bypass grafting (CABG) was released to the public. ${ }^{2}$ At both the individual surgeon and hospital level, those with reported low mortality rates saw rises in their market shares in the years following reporting and surgeons were able to charge more for their services. ${ }^{2,9}$ Thus, it follows that those listed with high mortality rates or with no data must have had a reduction in the overall patient marketplace. In fact, 1 study showed those in the lowest quartile of performance were 3.5 times more likely to stop practicing. ${ }^{9}$ Given the possibility of real financial and career fallout, more than $60 \%$ of cardiothoracic surgeons reported less willingness to operate on the sickest patients and had refused at least 1 ill patient in the prior calendar year because of fear of its effect on mortality rate. At the same time, patients who were candidates for aortic repair, which is not reported, with similar comorbidities as CABG candidates, were significantly more likely to receive operations. ${ }^{1,2}$ Reporting also encourages "gaming" of the statistics by exaggerating preoperative morbidity to change risk assessments, changing case classification to fall outside of the reported case category, and transferring patients to long-term care facilities if a death is anticipated..$^{30}$ As distribution and penetration of online information and quality reports are not equal among all populations, 1 study showed after CABG reports were released, patients of higher socioeconomic classes were treated by surgeons with lower mortality rates while patients of lower socioeconomic classes were treated by surgeons with higher mortality rates. ${ }^{9}$ Furthermore, an unexpected consequence of reporting surgeon outcomes is decreased access to cases for trainees, as any complication would be reported under the primary surgeon's record. ${ }^{2,14,26}$ While outcome reporting has been shown to increase interest in quality improvement activities, there has been no study showing that these reports actually change surgeon mortality rates. ${ }^{2,9}$ Thus, despite the uncertain validity and meaning of reported surgeon outcomes, there can be certain financial, patient access, and training issues that follow.

\section{Other Possible Metrics}

Given the problems of validity with current publically available performance measures, the question remains: what would be an accurate way to provide surgeon feedback and help the public make informed choices? Any valid surgeon reporting would require accurate input data, accepted definitions and assessments of complications and risks, and adequate sample size. The National Surgical Quality Improvement Program (NSQIP) has been a curated data set collected by sampling patient outcomes of hospital systems and services and producing reports for private review, but not open to the public. This has been effective in improving outcomes of participating hospital systems, but given random sampling of procedures, does not aim to provide data on specific surgeons. In a study of whether these data could be extrapolated out to provide feedback to specific surgeons, the NSQIP was found to have recorded data on $22 \%-35 \%$ of any individual surgeon's case volumes and that disagreement in the data from the NSQIP and data from the total surgeon's case volume could vary by up to $29 \% .16$ Therefore, while the NSQIP does resolve the issue of quality of data, it does not completely eliminate the bias of small sample size and risk adjustment. Not surprisingly, most surgeons oppose the use of the NSQIP to produce surgeon-specific measures and do not support public release of these data. ${ }^{22}$ Alternatively, it perhaps is more logical to focus on hospital-based metrics rather than individual statistics. ${ }^{3,31}$ Individual surgeon statistics falsely attribute outcome to a surgeon alone, whereas the real outcome for a patient relies heavily on hospital, staff, and resources. Attempting to rank individual surgeons may remain futile as outliers remain rare 
and the real statistical difference between surgeons may, in fact, not be as significant as the public may assume. ${ }^{30}$

\section{Conclusions}

The goals of transparency in reporting outcomes may be important in helping patients attain a high quality of care from surgeons. For these data to be valid, however, the approach must include high-quality data input, accepted definitions of complication and risk, and adequate sample size. The Consumers' Checkbook and ProPublica surgeon rating websites harbor severe methodological issues with all of the basic necessities for valid reporting. A substantial number of spinal neurosurgeons and orthopedic surgeons from top-ranked hospitals who specialize in spine, hip, or knee surgery have no ratings on either site or inconsistent data. Though Consumers' Checkbook and ProPublica evaluate the same surgeons, we found minimal correlation between the 2 websites in how surgeons were graded. Misusing administrative data to adversely impugn individual physicians, based upon poorly executed analysis, is not constructive and aids neither patients nor physicians.

\section{Acknowledgments}

This work was supported by a Congress of State Neurological Surgeons Socioeconomic Fellowship to Dr. Xu.

\section{References}

1. Burack JH, Impellizzeri P, Homel P, Cunningham JN Jr: Public reporting of surgical mortality: a survey of New York State cardiothoracic surgeons. Ann Thorac Surg 68:11951202, 1999

2. Burns EM, Pettengell C, Athanasiou T, Darzi A: Understanding the strengths and weaknesses of public reporting of surgeon-specific outcome data. Health Aff (Millwood) 35:415-421, 2016

3. Campbell PG, Malone J, Yadla S, Maltenfort MG, Harrop JS, Sharan AD, et al: Early complications related to approach in thoracic and lumbar spine surgery: a single center prospective study. World Neurosurg 73:395-401, 2010

4. Consumers' Checkbook: More About Our Measures of Outcomes. Washington, DC: Consumers Checkbook, 2015 (http://www.checkbook.org/surgeonratings/?action=article\& articleID=more-about-our-measures-of-outcomes) [Accessed July 26, 2016]

5. Consumers' Checkbook: Technical Appendix for Outcome Measures. Washington, DC: Consumers' Checkbook, 2015 (http://www.checkbook.org/surgeonratings/articles/ technical\%20appendix.pdf) [Accessed July 26, 2016]

6. Faciszewski T, Winter RB, Lonstein JE, Denis F, Johnson L: The surgical and medical perioperative complications of anterior spinal fusion surgery in the thoracic and lumbar spine in adults. A review of 1223 procedures. Spine (Phila Pa 1976) 20:1592-1599, 1995

7. Fountas KN, Kapsalaki EZ, Nikolakakos LG, Smisson HF, Johnston KW, Grigorian AA, et al: Anterior cervical discectomy and fusion associated complications. Spine (Phila Pa 1976) 32:2310-2317, 2007

8. Friedberg MW, Pronovost PJ, Shahian DM, Safran DG, Bilimoria KY, Elliot MN, et al: A Methodological Critique of the ProPublica Surgeon Scorecard. Santa Monica, CA: RAND Corporation, 2015 (http://www.rand.org/pubs/ perspectives/PE170.html) [Accessed July 26, 2016]

9. Fung CH, Lim YW, Mattke S, Damberg C, Shekelle PG:
Systematic review: the evidence that publishing patient care performance data improves quality of care. Ann Intern Med 148:111-123, 2008

10. Goldhahn S, Sawaguchi T, Audigé L, Mundi R, Hanson B, Bhandari M, et al: Complication reporting in orthopaedic trials. A systematic review of randomized controlled trials. J Bone Joint Surg Am 91:1847-1853, 2009

11. Hassan BK, Sahlström A, Dessau RB: Risk factors for renal dysfunction after total hip joint replacement; a retrospective cohort study. J Orthop Surg 10:158, 2015

12. Kalanithi PS, Patil CG, Boakye M: National complication rates and disposition after posterior lumbar fusion for acquired spondylolisthesis. Spine (Phila Pa 1976) 34:19631969, 2009

13. Katz JN, Barrett J, Mahomed NN, Baron JA, Wright RJ, Losina E: Association between hospital and surgeon procedure volume and the outcomes of total knee replacement. J Bone Joint Surg Am 86-A:1909-1916, 2004

14. Khan OA, Iyengar S, Pontefract DE, Rogers V, Ohri SK, Livesey SA: Impact of surgeon-specific data reporting on surgical training. Ann R Coll Surg Engl 89:796-798, 2007

15. Kreder HJ, Deyo RA, Koepsell T, Swiontkowski MF, Kreuter W: Relationship between the volume of total hip replacements performed by providers and the rates of postoperative complications in the state of Washington. J Bone Joint Surg Am 79:485-494, 1997

16. Kuhnen AH, Marcello PW, Roberts PL, Read TE, Schoetz DJ, Rusin LC, et al: Can the National Surgical Quality Improvement Program provide surgeon-specific outcomes? Dis Colon Rectum 58:247-253, 2015

17. Lebude B, Yadla S, Albert T, Anderson DG, Harrop JS, Hilibrand A, et al: Defining "complications" in spine surgery: neurosurgery and orthopedic spine surgeons' survey. J Spinal Disord Tech 23:493-500, 2010

18. Lee HB, Mears SC, Rosenberg PB, Leoutsakos JM, Gottschalk A, Sieber FE: Predisposing factors for postoperative delirium after hip fracture repair in individuals with and without dementia. J Am Geriatr Soc 59:2306-2313, 2011

19. Mahomed NN, Barrett JA, Katz JN, Phillips CB, Losina E, Lew RA, et al: Rates and outcomes of primary and revision total hip replacement in the United States Medicare population. J Bone Joint Surg Am 85-A:27-32, 2003

20. Memtsoudis SG, Ma Y, González Della Valle A, Mazumdar M, Gaber-Baylis LK, MacKenzie CR, et al: Perioperative outcomes after unilateral and bilateral total knee arthroplasty. Anesthesiology 111:1206-1216, 2009

21. Nasser R, Yadla S, Maltenfort MG, Harrop JS, Anderson DG, Vaccaro AR, et al: Complications in spine surgery. J Neurosurg Spine 13:144-157, 2010

22. Neuman HB, Michelassi F, Turner JW, Bass BL: Surrounded by quality metrics: what do surgeons think of ACS-NSQIP? Surgery 145:27-33, 2009

23. Peter WF, Dekker J, Tilbury C, Tordoir RL, Verdegaal SH, Onstenk R, et al: The association between comorbidities and pain, physical function and quality of life following hip and knee arthroplasty. Rheumatol Int 35:1233-1241, 2015

24. Pierce O, Allen M: Assessing Surgeon-Level Risk of Patient Harm During Elective Surgery for Public Reporting. New York: ProPublica, 2015. (https://static. propublica.org/projects/patient-safety/methodology/surgeonlevel-risk-methodology.pdf) [Accessed July 26, 2016]

25. Pierce O, Allen M: How we measured surgical complications. ProPublica. July 14, 2015. (https://www.propublica.org/ article/surgeon-level-risk-short-methodology) [Accessed July 26, 2016]

26. Radford PD, Derbyshire LF, Shalhoub J, Fitzgerald JE: Publication of surgeon specific outcome data: a review of implementation, controversies and the potential impact on surgical training. Int J Surg 13:211-216, 2015 
27. Ratliff JK, Lebude B, Albert T, Anene-Maidoh T, Anderson G, Dagostino P, et al: Complications in spinal surgery: comparative survey of spine surgeons and patients who underwent spinal surgery. J Neurosurg Spine 10:578-584, 2009

28. Roche JJ, Wenn RT, Sahota O, Moran CG: Effect of comorbidities and postoperative complications on mortality after hip fracture in elderly people: prospective observational cohort study. BMJ 331:1374, 2005

29. Schoenfeld AJ, Ochoa LM, Bader JO, Belmont PJ Jr: Risk factors for immediate postoperative complications and mortality following spine surgery: a study of 3475 patients from the National Surgical Quality Improvement Program. J Bone Joint Surg Am 93:1577-1582, 2011

30. Shahian DM, Normand SL, Torchiana DF, Lewis SM, Pastore JO, Kuntz RE, et al: Cardiac surgery report cards: comprehensive review and statistical critique. Ann Thorac Surg 72:2155-2168, 2001

31. Sherman KL, Gordon EJ, Mahvi DM, Chung J, Bentrem DJ, Holl JL, et al: Surgeons' perceptions of public reporting of hospital and individual surgeon quality. Med Care 51:10691075,2013

32. Talmo CT, Aghazadeh M, Bono JV: Perioperative complications following total joint replacement. Clin Geriatr Med 28:471-487, 2012

33. van Walraven C, Austin PC, Jennings A, Quan H, Forster AJ: A modification of the Elixhauser comorbidity measures into a point system for hospital death using administrative data. Med Care 47:626-633, 2009

34. Veeravagu A, Cole TS, Azad TD, Ratliff JK: Improved capture of adverse events after spinal surgery procedures with a longitudinal administrative database. J Neurosurg Spine 23:374-382, 2015

35. Villavicencio AT, Burneikiene S, Bulsara KR, Thramann JJ: Perioperative complications in transforaminal lumbar interbody fusion versus anterior-posterior reconstruction for lumbar disc degeneration and instability. J Spinal Disord Tech 19:92-97, 2006

36. Whitmore RG, Stephen JH, Vernick C, Campbell PG, Yadla S, Ghobrial GM, et al: ASA grade and Charlson Comorbidity Index of spinal surgery patients: correlation with complications and societal costs. Spine J 14:31-38, 2014
37. Yadla S, Malone J, Campbell PG, Maltenfort MG, Harrop JS, Sharan AD, et al: Early complications in spine surgery and relation to preoperative diagnosis: a single-center prospective study. J Neurosurg Spine 13:360-366, 2010

38. Yadla S, Malone J, Campbell PG, Nasser R, Maltenfort MG, Harrop JS, et al: Incidence of early complications in cervical spine surgery and relation to preoperative diagnosis: a singlecenter prospective study. J Spinal Disord Tech 24:50-54, 2011

39. Yli-Kyyny T, Ojanperä J, Venesmaa P, Kettunen J, Miettinen $\mathrm{H}$, Salo J, et al: Perioperative complications after cemented or uncemented hemiarthroplasty in hip fracture patients. Scand J Surg 102:124-128, 2013

\section{Disclosures}

Dr. Veeravagu has direct stock ownership in Precision Oncology.

\section{Author Contributions}

Conception and design: Ratliff. Acquisition of data: $\mathrm{Xu}, \mathrm{Li}$, Swinney. Analysis and interpretation of data: Xu. Drafting the article: $\mathrm{Xu}, \mathrm{Li}$. Critically revising the article: all authors. Reviewed submitted version of manuscript: all authors. Approved the final version of the manuscript on behalf of all authors: Ratliff. Statistical analysis: Xu. Study supervision: Ratliff.

\section{Supplemental Information}

Previous Presentations

Portions of this work were presented in abstract form as proceedings at the AANS Annual Scientific Meeting in Chicago, Illinois, on May 2, 2016.

\section{Correspondence}

John Ratliff, Department of Neurosurgery, Stanford University School of Medicine, 300 Pasteur Dr., Stanford, CA 94305-5327. email: jratliff@stanford.edu. 